Correspondence

\title{
Treatments for depression: Side-effects, adverse events and health risks
}

\section{Kirsch ${ }^{1}$, AR Ness ${ }^{2}$, KM Appleton ${ }^{3 *}$}

${ }^{1}$ Program in Placebo Studies, Harvard Medical School, Boston, US.

${ }^{2}$ NIHR Bristol Biomedical Research Centre, University of Bristol, Bristol, UK.

\section{${ }^{3}$ Research Centre for Behaviour Change, Psychology Department, Bournemouth University,} Bournemouth, UK.

*Corresponding author: Prof. Katherine Appleton, email: k.appleton@bournemouth.ac.uk

Debates over the benefits of n-3 long-chain polyunsaturated fatty acids ( $n$-3PUFAs) or omega-3 oils for depression continue. Meta-analyses report small but statistically significant benefits compared to placebo (Appleton et al., 2015; Bai et al., 2018; Grosso et al., 2014; Lin et al., 2012), but the clinical significance of any reported benefit has not been adequately considered.

Clinical significance requires effect sizes that are large enough to produce important clinical improvements. In the UK, the National Institute for Health and Care Excellence (NICE, 2009) uses a standardized mean difference (SMD) between drug and placebo of 0.50 or a difference of 3 points on the Hamilton Rating Scale for Depression (HRSD) as cutoffs for clinical significance in the treatment of depression. However, in a patient-level meta-analysis by Leucht et al. (2013), a 3-point difference on the HRSD corresponded to a Clinical Global Impression of Improvement (CGI-I) rating of "no change". Minimal improvement on the CGI-I corresponded to an HRSD change of 7 points or an SMD of 0.88 (Moncreiff \& Kirsch, 2005).

Effect sizes reported in reviews of $n$-3PUFAs as a treatment for depression are small to moderate (Appleton et al., 2015; Bai et al., 2018; Grosso et al., 2014; Lin et al., 2012), with 95\% confidence intervals that are typically wide, suggesting low reliability (e.g., Appleton et al., 2015: SMD $=0.30$ (95\%Cls 0.10, 0.50), Bai et al., 2018 (>1.5g/d n-3PUFA): SMD=0.43 (95\%Cls 0.04, 0.82); Grosso et al., 2014: $S M D=0.56$ (95\%Cls 0.20, 0.92); Lin et al., 2012: SMD=0.29 (95\%Cls 0.10, 0.48)). Without even considering a likely overestimation of benefits (Appleton et al., 2015), only one of these effect sizes 
meets the NICE criteria for clinical significance or comes close to the SMD corresponding to a minimal difference in CGI-I ratings. These small and unreliable effect sizes for n-3PUFAs indicate that they may not be a good choice for the treatment of depression (Appleton et al., 2015).

To investigate these effect sizes further, we have calculated the improvement for n-3PUFAs and placebo in studies using the HRSD that were included in the Appleton et al. (2015) meta-analysis. These analyses reveal a weighted mean improvement (WMI) of 9.75 for n-3PUFAs versus 8.00 for placebo, a clinically unimportant difference of 1.75 points on the HRSD. However, additional analyses demonstrate clinically meaningful improvements in both n-3PUFA and placebo groups from study start to study end - effect sizes of 1.72 and 1.31, respectively. A caution should be added to these findings, due to likely bias as above, but these findings suggest that n-3PUFAs can produce clinically significant improvements, but that these improvements may largely be due to a placebo response.

Small effect sizes in studies using n-3PUFAs versus placebo are comparable to those reported in metaanalyses of antidepressants (e.g. Kirsch et al., 2008: SMD=0.32 (95\%Cls 0.25, 0.40), Cipriani et al., 2018 : $\mathrm{SMD}=0.30(95 \% \mathrm{Cls} 0.26,0.34))$, although the effects for antidepressants are more likely to be robust, as confidence intervals are narrower. The differences in WMI on the HRSD found for antidepressants versus placebo are also comparable those of n-3PUFAs (e.g., Kirsch et al., 2008: WMI=1.80, Fournier et al., 2010: WMI=1.94, Stone et al., 2018: WMI=1.80), as are the within-group effect sizes. Kirsch et al. (2008) reported within-group improvements (from study start to study end) of 1.24 for antidepressants and 0.92 for placebo, and these benefits are not obtained in wait-list or supportive care control groups (Khan et al., 2012; Kirsch et al., 1998; Leuchter et al., 2014). Khan et al. (2012) compared the response to various treatments for depression and found all treatments to be equally effective and only slightly better than placebo or standard care. The percent improvement was $46 \%$ for antidepressants and $38 \%$ for placebo. Similarly, the data reported by Appleton et al. (2015) show 44\% improvement for n-3PUFAs and $35 \%$ for placebo.

How then are we to decide between treatments? When the evidence does not warrant recommending a particular treatment on the basis of differences in efficacy, consideration of differences in side effects, adverse events and health risks is recommended (Gartlehner et al., 2011). We make no recommendations for treatment based on our analyses, but direct comparisons of the benefits and risks of n-3PUFAs, placebo and antidepressants would be of value. 


\section{References}

Appleton, K.M., Sallis, H.M., Perry, R., Ness, A.R., Churchill, R. 2015. Omega-3 fatty acids for depression in adults. Cochrane Database Syst. Rev. 11, CD004692.

Bai, Z.-G., Bo, A., Wu, S.-J., Gai, Q.-Y., Chi, I. 2018. Omega-3 polyunsaturated fatty acids and reduction of depressive symptoms in older adults: A systematic review and meta-analysis. J. Affect. Disord. 241, 241248.

Cipriani, A., Furukawa, T.A., Salanti, G., et al. 2018. Comparative efficacy and acceptability of 21 antidepressant drugs for the acute treatment of adults with major depressive disorder: A systematic review and network meta-analysis. Lancet 391, 10128, 1357-66.

Fournier, J.C., DeRubies, R.J., Hollon, S.D., Dimidjian, S., Amsterdam, J.D., Shelton, R.C., Fawcett, J. 2010. Antidepressant drug effects and depression severity: A patient-level meta-analysis. J. Am. Med. Assoc. 303, 47-53.

Gartlehner, G., Hansen, R.A., Morgan, L.C., et al. 2011. Comparative benefits and harms of secondgeneration antidepressants for treating major depressive disorder: An updated meta-analysis. Ann. Intern. Med. 155, 772-85.

Grosso, G., Pajak, A., Marventano, S., et al. 2014. Role of omega-3 fatty acids in the treatment of depressive disorders: a comprehensive meta-analysis of randomized clinical trials. PLoS One 9, e96905.

Khan, A., Faucett, J., Lichtenberg, P., Kirsch, I., Brown, W.A. 2012. A systematic review of comparative efficacy of treatments and controls for depression. Plos One 7, e41778.

Kirsch, I., Deacon, B.J., Huedo-Medina, T.B., et al. 2008. Initial severity and antidepressant benefits: a meta-analysis of data submitted to the Food and Drug Administration. PLoS Med. 5, e45.

Kirsch, I., Sapirstein, G. 1998. Listening to Prozac but hearing placebo: A meta-analysis of antidepressant medication. Prevention and Treatment, Article 0002a. 
92 Leucht, S., Fenneema, H., Engel, R., Kaspers-Janssen, M., Lepping, P., Szegedi, A. 2013. What does the 93 HAMD mean? J. Affect. Disord. 148, 243-8.

94

95 Leuchter, A.F., Hunter, A.M., Tartter, M., Cook, I.A. 2014. Role of pill-taking, expectation and therapeutic 96 alliance in the placebo response in clinical trials for major depression. Br. J. Psychiatry 205, 443-9.

97

98 Lin, P.Y., Mischoulon, D., Freeman, M.P., et al. 2012. Are omega-3 fatty acids anti-depressants or just 99 mood-improving agents? The effect depends upon diagnosis, supplement preparation, and severity of

101

Moncreiff, J., Kirsch, I. 2005. Efficacy of antidepressants in adults. Brit. Med. J. 16, 331(7509), 155-7.

103

104 depression. Mol. Psychiatry 17, 1161-3.

National Institute for Clinical Excellence. 2009. Depression: management of depression in primary and

107 Stone, M., Kalaria, S., Richardville, K., Miller, B. 2018. Components and Trends in Treatment Effects in 108 Randomized Placebo-controlled Trials in Major Depressive Disorder from 1979-2016. Paper presented at 109 the American Society of Clinical Psychopharmacology, Miami, 2018.

110

111 Turner, E.H., Matthews, A.M., Linardatos, E., et al. 2008. Selective publication of antidepressant trials 112 and its influence on apparent efficacy. N. Engl. J. Med. 358, 252-60. 